among overseas-born males predominantly from countries with a bivalent vaccine programme, suggests these men receive herd protection for 16/18 from their vaccinated female partners in their countries of origin.

\section{LOW PROPORTION OF MEN WHO HAVE SEX WITH MEN (MSM) TESTED FOR HEPATITIS C DESPITE HIGH PREVALENCE IN 2 GENITO-URINARY MEDICINE (GUM) CLINICS}

Thomas Pasvol*, Palwasha Khan, Arun Thiagarajan, Subathira Dakshina, Liat Sarner, Chloe Orkin. Bart's Health NHS Trust, London, UK

10.1136/sextrans-2016-052718.27

Background/introduction Screening for HIV and hepatitis B (HBV) is recommended for MSM attending GUM clinics. Hepatitis $\mathrm{C}$ testing is recommended for all HIV positive MSM. However, the PROUD pilot study reported an HCV incidence of $3.1 \%$ in high-risk, HIV negative MSM.

Aim(s)/objectives To report on proportion tested and prevalence of blood-borne viruses (BBVs) amongst MSM attending GUM clinics

Methods We collected demographic data and numbers tested for BBVs in all MSM attending 2 GUM clinics between 01/07/14 and 30/06/15 from electronic records. We compared proportion tested and prevalence in high-risk vs low-risk MSM and in HIV + vs HIV- MSM. High-risk was defined as $\geq 1$ sexually transmitted Infection (STI) i.e. Gonorrhoea, chlamydia and syphilis.

Results 4,415 patients were included. 3,289 (88.0\%) were tested for HIV, 2,162 (49.1\%) for HBV, 794 (18.1\%) for HCV. Positives: 48 (1.5\%) HIV, 11 (0.5\%) HBV and 18 (2.3\%) HCV [9/16 $(56.3 \%)$ viraemic]. 1,003 $(22.7 \%)$ were diagnosed with an STI: Syphilis 159 (3.6\%), Gonorrhoea 640 (14.5\%), Chlamydia 398 (9.0\%). BBV prevalence was higher in high-risk vs low-risk MSM: HIV $23(3.4 \%)$ vs 25 (1.0\%); HBV 4 (0.7\%) vs 7 $(0.4 \%)$; HCV $10(3.5 \%)$ vs 8 (1.6\%).More HIV+ MSM were tested for HCV; 198 (27.8\%) vs 587 (16.9\%) HIV- (crude OR 1.9 (95\% CI 1.6-2.3). HCV prevalence in those tested was 12 $(6.1 \%)$ in HIV+ and 6 (1.0\%) in HIV-.

Discussion/conclusion MSM were less likely to be tested for HCV than for HIV. Amongst those tested, HCV prevalence was $5 \mathrm{x}$ the national prevalence $(0.4 \%)$. Prevalence of viral hepatitis was highest in HIV+ and in high-risk MSM suggesting that testing efforts should be increased.

\section{O029 SEXUAL FUNCTION PROBLEMS IN BRITISH 16-21 YEAR OLDS: CAUSE FOR CONCERN?}

\footnotetext{
${ }^{1,2}$ Kirstin Mitchell* ${ }^{3}$ Rebecca Geary, ${ }^{4}$ Cynthia Graham, ${ }^{3}$ Soazig Clifton, ${ }^{3}$ Catherine Mercer, ${ }^{2}$ Ruth Lewis, ${ }^{2}$ Wendy Macdowall, ${ }^{2}$ Jessica Datta, ${ }^{3}$ Anne Johnson, ${ }^{2}$ Kaye Wellings. ${ }^{1}$ University of Glasgow, Glasgow, Scotland, UK; ${ }^{2}$ London School of Hygiene and Tropical Medicine, London, UK; ${ }^{3}$ University College London, London, UK; ${ }^{4}$ University of Southampton, Southampton, UK
}

10.1136/sextrans-2016-052718.28

Background/introduction Sexual function is largely absent from the policy discourse on young people's sexual health. The omission is troubling, given the link between low sexual function and indicators of risk (including higher partner numbers, paying for sex, non-consensual sex and STI diagnosis). An absence of data permits this silence.
Aim(s)/objectives To address the gap in empirical data on sexual function problems in young people aged 16 to 21 in Britain.

Methods Descriptive statistics from a national probability survey of 15,162 British men and women (Natsal-3), undertaken from 2010-2012 using computer-assisted self-interviews (CASI). Complex survey analyses of data from participants aged 16-21 (854 men and 1021 women sexually active in the last year; 262 men and 255 women sexually experienced but not active in the last year).

Results Distressing sexual function problems ( $>3$ months in last year) were reported by $9.1 \%$ of men and $13.4 \%$ of women. Most common among men was reaching a climax too quickly $(4.5 \%)$ and among women, difficulty reaching climax at all $(6.3 \%)$. The majority of young people experiencing problems did not seek help, and those that did rarely sought out professionals. Around $6 \%$ of those currently sexually active, and $10 \%$ of those not so, reported avoiding sex because of sexual function problems.

Discussion/conclusion Sexual function problems are common among young people and are largely unaddressed. Addressing these clear needs will have benefits for other aspects of sexual health. Reassurance in clinical settings and information/advice in educational settings are inexpensive and potentially effective strategies.

\section{SEXUAL AND REPRODUCTIVE HEALTH CONSULTATIONS IN A NGO PRIMARY CARE FACILITY OVER A NINE WEEK PERIOD}

${ }^{1}$ Charline Bradshaw*, ${ }^{*}$ Aliza Amlani, ${ }^{1}$ Fionnuala Finnerty, ${ }^{1,2}$ Daniel Richardson. ${ }^{1}$ Brighton \& Sussex University Hospitals Trust, Brighton, UK; ${ }^{2}$ Brighton \& Sussex Medical School, Brighton, UK

\subsection{6/sextrans-2016-052718.29}

Background/introduction The emergence of the "jungle" camp in Calais has been described as a humanitarian emergency. There are internationally recognised minimum standards for provision (MISP) of sexual and reproductive health (SRH) care in a crisis situation. It has been reported that the Calais "jungle" camp has not met these.

Methods We reviewed clinic attendances/consultations during a 9 week period, from mid-December 2015 to February 2016, at a non-governmental organisation (NGO) primary care clinic in the Calais "jungle" staffed by volunteer clinicians

Results 394 women and 6118 men aged 15-44 attended the primary care clinic during the study period. Of these, 22 men $(0.4 \%)$ and 39 women (10\%) women sought a consultation regarding SRH. There were 17 requests for pregnancy tests $(1.8$ per week), 9 termination of pregnancy requests (1 per week) and 2 consultations where sexual violence in women was disclosed, (0.7 per week). 22/6118 men (0.4\%) sought advice or treatment for a sexually transmitted infection during the study period.

Discussion/Conclusion Provision of (sexual) \& reproductive health in Calais is limited, however our preliminary data shows that demand is high: men do not access the service leaving women particularly vulnerable to poor sexual health and possibly violence. The data is likely to represent the tip of an iceberg. Given the extent of the current refugee crisis and the increase in transit camps around Europe, lessons need to be learned from the Calais "jungle" camp. 